\title{
REQUIREMENTS FOR TRANSITIONING BUSINESS PROCESS SIMULATION MODELS TO REAL-TIME OPERATIONAL SYSTEMS
}

\author{
Peter Floss \\ Gensym Corporation \\ 125 CambridgePark Drive \\ Cambridge, MA 02140, U.S.A.
}

\begin{abstract}
The value of modeling and simulating business processes has recently received increased attention with the interest in business process re-engineering (BPR). Many re-engineering projects are done in conjunction with major system implementation projects, which can also benefit from modeling and simulation. As these system implementation projects become operational, the simulation models used to design and analyze them, and their associated business processes, have typically become obsolete or seen limited use. Significant additional value can be realized if these same models can become part of the operational systems being implemented, either as executive business monitoring tools or as business workflow control tools. This paper describes the characteristics of these real-time operational systems and the requirements for modeling and simulation tools that support transitioning to these types of systems. A purchase order request application is then presented as a concrete example of a real-time operational system.
\end{abstract}

\section{INTRODUCTION}

The emphasis within organizations to become more process oriented and to focus on core business processes (Hammer 1996) has led to an increased interest in modeling and simulating business processes. The need for modeling of business processes quite often becomes apparent when organizations embark upon large system implementation projects. Project teams use models to document and benchmark existing processes, in "As Is" models. "Vision" models are used to reach consensus on and communicate high-level, long-term principles and objectives within large project teams. Actual proposed process and system changes are represented by "To Be" models, which emphasize manageable implementation and change management projects. The value of models at each of these project stages is increasingly becoming accepted, as are the benefits of also simulating these models.

As these large system implementation projects move from design into development, testing, and implementation, the models used to design and analyze the systems and processes see increasingly limited use. Significant additional value can be realized if these same models can become part of the operational systems being implemented. This allows the operational processes to be monitored and/or controlled by the same model used for its design. These real-time operational systems are a result of the integration of modeling and simulation tools with real-time deployment environments.

\section{REAL-TIME OPERATIONAL SYSTEMS}

The tight integration of business process modeling and simulation tools with real-time deployment environments offers the potential for significant additional value to the end user organization. Models that were previously used for the design and analysis of business processes can now also actively participate in the operation of the business. This allows for better management decision making and improved processes based upon more timely information about the performance of the business.

An important aspect of these systems is the tight integration between the modeling tool and the real-time environment. An alternative approach consisting of translating the model to a form that is usable by some other environment (e.g., a workflow engine) is still useful, but can not provide all of the same benefits. The translation process introduces maintenance issues and does not support working within a single environment. The key to the tight integration is that the actual model becomes a part of the operational systems, not a clone or translation of the model.

These real-time operational systems can be separated into at least two high-level categories, depending upon their role in the actual execution of the business 
processes. The first is a relatively passive role, where the real-time operational systems are used to monitor the performance of key metrics within the business processes. These types of systems are used to provide high-level, enterprise-wide views into the performance of the organization. The second category consists of realtime operational systems that actively participate in the actual execution of the processes. They are actively involved in intelligently routing and controlling the flow of work within the business processes.

\subsection{Executive Business Monitoring}

An executive business monitoring application, based upon a model used to design and simulate the process, allows that same model to be used to view the ongoing performance of that business process. It allows management to navigate process views of their business and review key metrics based upon the real-time data describing the performance of the business. For example, selecting the order entry task within an order management model could bring up key metrics such as the current number of orders waiting to be entered and the average time to enter an order. Identifying real-time bottlenecks and then dynamically reassigning resources is an example of how management might use this type of application to make better decisions about the operation of their business.

In addition to monitoring the execution of a business, the tight integration of the modeling and simulation environment provides the opportunity to simulate forward from the current state of the business. This allows managers to predict the short-term performance of the organization based upon the actual real-time current state of the business. Estimating the staffing requirements for the next several days based upon the current work in progress is an example of how this forward simulation capability might be used.

\subsection{Business Workflow Control}

Real-time operational systems can also participate more actively in the execution of the business processes through a workflow control application. The same model that was used to design and simulate the business processes is now used in routing and controlling the overall system. The actual execution of the workflow can take many different forms including dispatching work to manual processes, interacting with legacy systems, invoking end-user applications, and interacting with databases. A high-level workflow control application might even utilize a workflow execution engine for the low-level execution of the work. The value of doing this in a dynamic real-time deployment environment is that changes can be instantly propagated to the actual business process. These changes can be as simple as changing a decision parameter and as complex as actually rerouting the work based upon the current state of the system. These changes can also be simulated prior to implementation in a separate version of the operational environment.

\section{TOOL REQUIREMENTS}

These real-time operational systems are possible because of the tight integration between the modeling and simulation tool and the real-time deployment environment. This integrated tool set requires certain major capabilities that are not necessarily found in traditional modeling and simulation tools. Some of these capabilities are provided directly by the real-time deployment environment and others require software to specifically integrate the features of the individual products. These major capabilities include synchronization with a real-time clock, event scheduling based upon external events, connectivity to external systems, operating within a distributed environment, an extensible modeling environment, and the ability to initialize a model to a predefined state.

\subsection{Real-Time Clock}

At the core of a discrete-event simulation tool is a simulation calendar, used to manage the events to be executed. When transitioning a simulation model to a real-time operational system, this simulation calendar must be synchronized with a real-time clock. When the simulation clock is synchronized, simulation time is incremented at a regular interval of real-time (e.g., every second) by that amount of time. One second of simulation time then takes exactly one second of realtime.

\subsection{Event Scheduling}

The completion of an activity within a discrete event simulation is typically scheduled based upon some duration specified within the model. An issue related to the real-time clock is that event scheduling must be handled differently with activities whose duration is determined by some external action (e.g., a user performing a task). One way to implement this is to schedule the event for some arbitrarily long duration (known to be longer than the actual duration) and then reschedule it to the current time when the external action completes. This external action must signal its completion to the simulation, for example, via a callback. 


\subsection{Connectivity}

Real world business systems consist of a wide variety of different technologies and systems. Examples include legacy systems, new applications, databases, ERP systems, and internet/intranet-based solutions. Real-time operational systems must therefore be able to connect to and communicate with a wide variety of different applications. This connectivity mechanism into and out of the simulation and real-time deployment environment must be quite flexible. It should ideally support both general-purpose connectivity solutions (which can be shared across many applications) and application specific connectivity solutions. Database integration is an example of a general-purpose connectivity solution that can be shared across multiple applications.

\subsection{Distributed Environment}

The systems that operate real world business processes are typically distributed across multiple computers. Closely related to connectivity is the capability to operate within a distributed environment. This can manifest itself in a wide variety of different forms. For example, a specific real-time operational application may call for monitoring business processes from a client system connected to a central server system. Another application may require the real-time operational system to actually be distributed across a number of different computers. A third application may simply need to communicate with an external system (e.g., a legacy system) on a different computer. All of these examples assume a networked environment with the capability to distribute portions of the real-time operational system across a number of computers.

\subsection{Extensible Environment}

Depending upon the application, real-time operational systems typically require modeling to a level of detail that is deeper than a simulation model intended to address a very specific question. Modeling environments that supports scripting or customizing complex portions of the model provide the necessary flexibility to achieve this level of detail. For example, a complex algorithm for routing work may require support beyond what can be provided directly by modeling and simulation tools. The most flexible environments for extending models provide a full programming language and complete set of development tools. This capability is also important in the transition to a real-time operational system, as some level of custom development is typically required.

\subsection{Model Initialization}

The ability to simulate forward from the current state of the business process requires that the model accurately reflect the current conditions. This can be accomplished by either keeping the real-time operational system synchronized with the business process (as would be the case in a business workflow control application) or by being able to initialize the model to a predefined state. In initializing the model, the current conditions would come from the actually executing business process, through the connectivity to the specific systems. The forward simulation could then help predict the shortterm behavior and requirements of the operational system, based upon these current conditions.

\section{EXAMPLE}

As previously mentioned, these real-time operational systems can take several different forms. As a concrete example of such a system, a purchase order request model is described as an example of a business workflow control application.

The model was first used to document and communicate the current process for requesting a purchase order for a particular product or service. The overall process is very much a manual one, consisting of getting signature approval from a variety of different managers within the organization (depending upon the value and type of purchase order being requested). The top-level view and one of the detail views of the model is shown in Figure 1. Opportunities for improving the process could be considered and evaluated using the simulation capabilities of the modeling tool.

This same model was transitioned to a business workflow control application that connects to several external systems. The system actually controls the routing of purchase order requests as they are acted upon (either approved, disapproved, or request more information) by the different managers. Requests for purchase orders are submitted via a custom client/serverbased application. The model contains a forward flow for requests that are in the process of being approved and a backward flow for requests that require more information or have been disapproved. The managers also interact with the purchase order requests via a custom client/server-based application that shows them a queue of requests awaiting their action. An electronic mail-based notification system is also useful for letting individuals know that actions have been performed on their requests or that several items are awaiting their action.

The purchase order requests are maintained within a database for persistent storage while they are being routed through the process. The actual purchase orders are generated by a financial system that is initiated by 


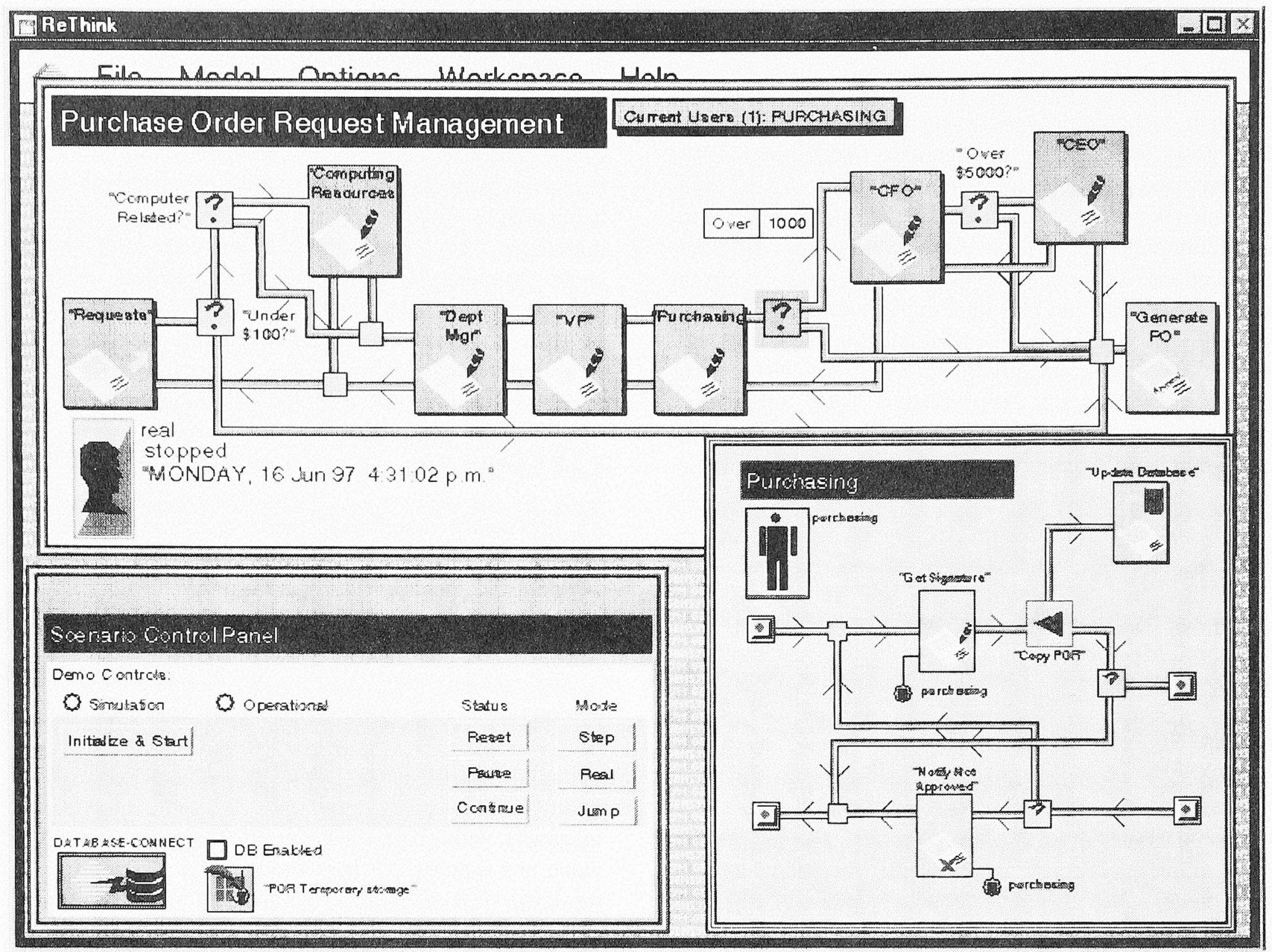

Figure 1: Purchase Order Request Application

this real-time-operational system after all of the approvals have been received.

One of the many very interesting benefits of this application is the ability to dynamically change the routing of the work. For example, the decision points before the routing to senior management for approval can be adjusted based upon the level of fiscal control required by current business pressures.

\section{ACKNOWLEDGMENTS}

This paper describes a vision for the design, simulation, and operational management of models within a single development and deployment environment. Many people within Gensym, and the ReThink consulting and development groups in particular, have contributed to this vision and the work presented in the example. The author gratefully acknowledges everyone at Gensym who has contributed directly or indirectly to the ideas presented in this paper.

\section{REFERENCES}

Hammer, M. 1996. Beyond Reengineering: How the Process-Centered Organization is Changing Our Work and Our Lives. HarperCollins Publishing.

\section{AUTHOR BIOGRAPHY}

PETER FLOSS is a senior consulting engineer in the Advanced Systems Business Unit of Gensym Corporation. He has over 9 years of professional software development experience. Since joining Gensym in May of 1995, he has led the development of the initial product release and major new version of ReThink $\circledast$, Gensym's business process modeling and simulation tool. Prior to joining Gensym, he was employed at Cambridge Technology Partners and at Digital Equipment Corporation. He holds Master of Science and Bachelor of Science degrees in Industrial Engineering from Purdue University. 